\title{
BMJ Open Midline incisional hernia prophylaxis using synthetic mesh in an emergency or urgent gastrointestinal tract surgery: a protocol for multicentre randomised clinical trial
}

\author{
Amarit Tansawet (D) ,, ${ }^{1,2}$ Pawin Numthavaj, ${ }^{2}$ Preeda Sumritpradit, ${ }^{3}$ \\ Suphakarn Techapongsatorn (D) , ${ }^{1}$ Gareth McKay (D) , John Attia (D) , \\ Ammarin Thakkinstian $^{2}$
}

To cite: Tansawet A, Numthavaj $P$, Sumritpradit $P$, et al. Midline incisional hernia prophylaxis using synthetic mesh in an emergency or urgent gastrointestinal tract surgery: a protocol for multicentre randomised clinical trial. BMJ Open 2021;11:e045541. doi:10.1136/ bmjopen-2020-045541

- Prepublication history and additional supplemental material for this paper are available online. To view these files, please visit the journal online (http://dx.doi.org/10.1136/ bmjopen-2020-045541).

Received 05 0ctober 2020 Accepted 13 August 2021
Check for updates

(c) Author(s) (or their employer(s)) 2021. Re-use permitted under CC BY-NC. No commercial re-use. See rights and permissions. Published by BMJ.

For numbered affiliations see end of article.

Correspondence to Dr Pawin Numthavaj; pawin.num@mahidol.ac.th

\section{ABSTRACT}

Introduction Between $5 \%$ and $30 \%$ of abdominal incisions eventually result in incisional hernias $(\mathrm{IHs})$ that can lead to severe complications and impaired quality of life. Unfortunately, IH repair is often unsuccessful; therefore, hernia prophylaxis is an important issue. The efficacy of mesh augmentation has been proven for hernia prophylaxis in high-risk patients, but no randomised clinical trial has evaluated prophylactic mesh placement in emergency/urgent gastrointestinal operations.

Methods and analysis A multicentre, prospective randomised, open and patient-assessor blinded endpoint design will be conducted. A total of 470 patients will be enrolled and randomly allocated to retrorectus mesh augmentation with lightweight polypropylene mesh or primary suture closure. The primary outcome is $\mathrm{IH}$ occurrence within 24 months of follow-up, while other clinical outcomes are secondary endpoints. A costeffectiveness analysis will be conducted from the societal and provider perspectives.

Ethics and dissemination Ethics approval was obtained from Ramathibodi Hospital (MURA2020/1478) and Vajira Hospital (COA164/2563). The protocol is on the process of submission to the local ethics committee of the other study sites. Results will be submitted for publication in a peer-reviewed journal.

Trial registration number TCTR20200924002.

\section{INTRODUCTION}

Incisional hernia (IH) is a common complication following an abdominal operation. The incidence of IH varies between $5 \%$ and $20 \%$ in the general population, increasing to almost $30 \%$ in high-risk populations; those with midline laparotomy for gastrointestinal (GI) surgery are the most vulnerable to IH. Evidence from meta-analyses suggests that mesh-augmented fascia closure may reduce IH incidence in midline incision, with a risk reduction of $70 \%-86 \% .^{2-7}$ Furthermore, a network meta-analysis ${ }^{8}$ has indicated that
Strengths and limitations of this study

- This study is one of only a few randomised clinical trials (RCTs) that will assess hernia prophylaxis in patients undergoing emergency/urgency gastrointestinal (Gl) surgery.

- The RCT will minimise selection bias and balance confounders that may affect incisional hernia occurrence.

- Cost-effectiveness analysis alongside this RCT will be performed to evaluate if the retrorectus mesh is cost-effective in emergency/urgency Gl surgery.

- A recruitment rate may be delayed with uncontrolled COVID-19 pandemic in Thailand situation.

mesh positioning in midline laparotomy also affects $\mathrm{IH}$ prophylaxis and that retrorectus mesh (RM) placement may be the most suitable option, considering both benefits and risks.

The incidence of $\mathrm{IH}$ ranges from 16\% to $33 \%$ after emergency laparotomy. ${ }^{9-13}$ A large retrospective study, consisting of 29739 subjects, reported that IH incidence after an emergency operation was as high as $16 \%$, compared with $2 \%$ following elective surgery. ${ }^{13}$ Nevertheless, prophylactic mesh efficacy in lowering hernia occurrence in emergency or urgency patients has never been evaluated in any randomised clinical trial (RCT). Evidence from observational studies $^{10}{ }^{11}$ comparing prophylactic mesh augmentation and conventional fascia closure in emergency abdominal operations highlights the benefits of mesh placement without significantly impacting complication rates. A recent meta-analysis ${ }^{14}$ to assess the effects of prophylactic mesh in emergency laparotomy has included only observational studies ${ }^{10} 11$ 
identifying significantly lower IH compared with nonprophylactic mesh. However, this evidence is potentially biased, given data synthesis is based on observational studies. A recent $\mathrm{RCT}^{15}$ just published on submission of our protocol has demonstrated efficacy of mesh prophylaxis in lower fascial dehiscence, not IH occurrence, when compared with no-mesh in emergency laparotomy.

Given the lack of evidence, an RCT is necessary to assess the benefits and risks of mesh for hernia prevention in patients undergoing midline laparotomy under emergency/urgency GI conditions. The proposed RCT will compare RM, using non-absorbable (polypropylene) mesh, to primary suture closure (PSC) to evaluate the hypothesis that RM will lower IH incidence compared with PSC in this particular group of patients. The primary endpoint is $\mathrm{IH}$, and other clinical outcomes will be considered.

\section{Study objectives}

\section{Primary objective}

This proposed study aimed to assess the IH prevention effect of the non-absorbable mesh when placed in the retrorectus plane compared with PSC.

\section{Secondary objectives}

- To assess the safety of mesh placement in terms of adverse events (eg, surgical site infection (SSI), seroma, haematoma, etc).

- To compare abdominal wall closure time, length of hospital stay, and level of postoperative pain between RM and PSC.

- To perform a cost-effectiveness analysis of RM augmentation in IH prophylaxis indication.

- To report the overall mesh removal rate.

\section{METHODS AND ANALYSIS}

\section{Study design and setting}

This study is a multicentre, prospective randomised, open, patient-assessor blinded endpoint trial, which will potentially include six centres: Ramathibodi, Vajira, Bhumibol Adulyadej, Hatyai, Maharat Nakhon Ratchasima and Surin hospitals. Protocol development follows the Standard Protocol Items: Recommendations for Interventional Trials guideline. ${ }^{16}$ This trial was registered at Thai Clinical Trials Registry.

\section{Patient and public involvement}

Patients or the public were not involved in the design, conduct, reporting or dissemination plans of our study.

\section{Inclusion criteria}

- Adults aged $\geq 18$ years.

- Have undergone any type of GI surgery within 24 hours after admission or consultation with suspected GI pathology.

- Have received midline abdominal incision with an incision length of at least one-fourth of the distance from the xiphoid process to the pubic symphysis.

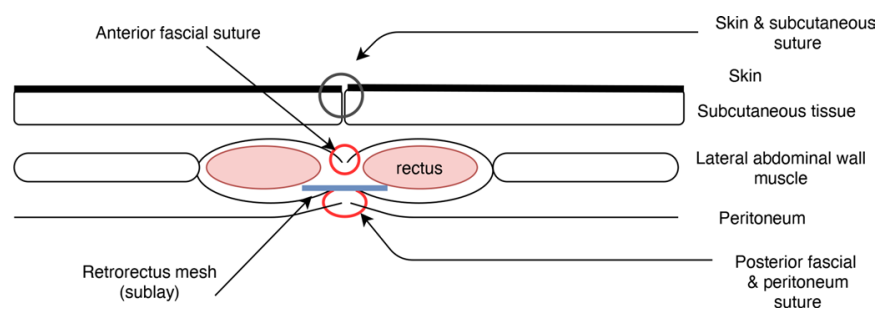

Figure 1 Retrorectus mesh augmentation.

- Have an American Society of Anesthesiologist physical status class of 1-4.

\section{Exclusion criteria}

Patients will be excluded if they have any of following criteria:

- Have any of following conditions:

Septic shock (haemodynamic instability).

Metastatic cancer (stage IV) at before or during operation.

Dirty surgical wound with severe contamination from faecal material and frank pus (surgical wound class 4). Massive devitalised bowel ischaemia.

Patients with following criteria will be ineligible:

- Potential second-look operation or planned revisionary surgery via a midline incision.

- Secondary fascial closure.

- Existence of midline IH and/or history of midline IH repair.

- Pregnancy or plan for pregnancy after surgery.

- Connective tissue disorders.

- Current or planned immunosuppressive use.

- Allergy to polypropylene.

\section{Intervention}

At the end of the index procedure, decontamination of the abdominal cavity will be performed with at least $3 \mathrm{~L}$ of normal saline. Participants will be allocated to either RM or PSC (figures 1 and 2) prior to abdominal wall closure. Intervention details will be as follows.

\section{Primary suture closure}

Single-layer closure of the linea alba will be performed for all collaborative sites using a 1-0 polydioxanone suture (PDS). Small tissue bite (ie, $5-8 \mathrm{~mm}$ bite) and small $(5 \mathrm{~mm})$ intersuture spacing with continuous suturing will be applied to ensure a wound to suture length ratio of 1:4; this will be recorded in a case record from. No retention suture will be applied. Additional decontamination with at least $1 \mathrm{~L}$ of normal saline will be performed

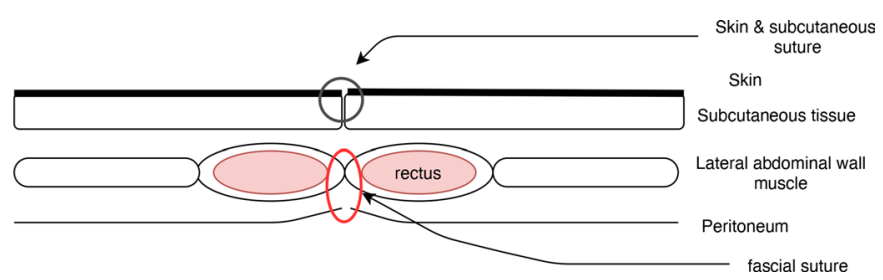

Figure 2 Primary suture closure. 
at the subcutaneous layer. Surgeons will decide whether skin and subcutaneous tissue will be closed or left open for wet dressing. In the event of skin and subcutaneous tissue closure, the subcutaneous space should be obliterated using multiple stitches of absorbable suture without a subcutaneous drain in place. Skin can be approximated using non-absorbable suture or staples.

\section{RM augmentation}

A plane between the rectus muscle and the posterior rectus fascia/peritoneum will be developed to achieve a $3 \mathrm{~cm}$ distance from the fascial edge in all directions. The posterior rectus fascia/peritoneum closure will be performed using a continuous suture with a 1-0 PDS. A piece of $6 \times 15 \mathrm{~cm}$ lightweight macroporous polypropylene mesh (weight $38 \mathrm{~g} / \mathrm{m}^{2}$ and $1.5 \mathrm{~mm}$ pore size) will be placed anterior to the posterior rectus fascia/peritoneum. Overlapping of the mesh and the fascia incision should be $3 \mathrm{~cm}$ in all directions. A second mesh piece can be used as necessary, with $2 \mathrm{~cm}$ overlapping with the first mesh piece. Mesh will be fixed to the posterior rectus fascia at its four corners and the mid-length using 3-0 polypropylene sutures. No drain will be placed in the retrorectus space. Closure of the anterior rectus fascia will be performed as described in the PSC group. Skin and subcutaneous tissue will be managed as described previously.

\section{Training}

Participating surgeons and surgical residents will be trained by principal investigators to achieve consistent standards for RM and PSC techniques. A tutorial video of RM and PSC is available for each study centre. For RCT enrolment, RM must have been performed on at least five occasions for each study site.

\section{Cointerventions}

Empirical antibiotics will be given according to suspected pathogens and allergic status for each participant. After culture and susceptibility results are available, the type of antibiotics and its course will be adjusted accordingly.
Medications will be administered intravenously and switched to oral form after being afebrile for 24-48 hours.

Participants will receive $0.5 \mathrm{mg} / \mathrm{kg}$ of pethidine or $0.05 \mathrm{mg} / \mathrm{kg}$ of morphine intravenously every 4 hours as a standard pain control with additional opioid doses for breakthrough pain. This pain control is initially for the first 24 hours and will be adjusted on subsequent days, depending on the participant's pain level. Other options for pain relief will include patient-controlled analgesia or epidural anaesthesia. After oral intake is resumed, oral acetaminophen or non-steroidal anti-inflammatory drugs will be prescribed.

An oral diet will be ordered when GI function is detected. Breathing exercises and rehabilitation will be encouraged as per usual practice. No abdominal binders will be applied.

\section{Outcomes and measurement \\ Primary endpoint}

IH occurrence is the primary outcome of interest and will be measured at 3, 6, 12, 18 and 24 months after index operation. A telephone call will be made by research assistants to remind participants of follow-up. The diagnosis will be made by physical examination. Dynamic ultrasound will be used as a supplement in equivocal cases (ie, participants who complain of lump or discomfort at a surgical incision without a definite physical sign of hernia). CT will only be considered where ultrasound results are uncertain.

\section{Secondary endpoints}

Table 1 shows the outcome measurements according to each follow-up visit. Secondary outcomes are listed as follows:

- SSI is defined according to the Centers for Disease Control and Prevention diagnostic criteria ${ }^{17}$ (see box 1). Both superficial and deep SSIs will be included. Because the intervention involves a foreign body placement, follow-up will be scheduled for 1 year to detect a deep SSI. Once there is SSI occurrence, treatment managements will be recorded including

Table 1 Timetable for clinical outcome measurements

\begin{tabular}{|c|c|c|c|c|c|c|c|}
\hline Outcome & $\begin{array}{l}\text { Days } 1 \\
\text { and } 3\end{array}$ & $\begin{array}{l}\text { Within } 1 \\
\text { month }\end{array}$ & 3 months & 6 months & 12 months & 18 months & 24 months \\
\hline Incisional hernia & & & $\checkmark$ & $\checkmark$ & $\checkmark$ & $\checkmark$ & $\checkmark$ \\
\hline Seroma & & $\checkmark$ & $\checkmark$ & & & & \\
\hline Burst abdomen & $\checkmark$ & $\checkmark$ & & & & & \\
\hline $\begin{array}{l}\text { Enterocutaneous fistula and } \\
\text { wound sinus }\end{array}$ & $\checkmark$ & $\checkmark$ & $\checkmark$ & $\checkmark$ & $\checkmark$ & & \\
\hline Acute pain & $\checkmark$ & & & & & & \\
\hline Chronic pain & & & $\checkmark$ & $\checkmark$ & $\checkmark$ & $\checkmark$ & $\checkmark$ \\
\hline
\end{tabular}


Box 1 Superficial and deep SSI according to the criteria of the Centers for Disease Control and Prevention

\section{Superficial SSI}

Infection occurs within 30 days after the operation and infection involves only skin or subcutaneous tissue of the incision and at least one of the following:

- Purulent drainage, with or without laboratory confirmation, from the superficial incision.

- Organisms isolated from an aseptically obtained culture of fluid or tissue from the superficial incision.

- At least one of the following signs or symptoms of infection: pain or tenderness, localised swelling, redness or heat, and superficial incision is deliberately opened by surgeon, unless incision is culture-negative.

- Diagnosis of superficial incisional SSI by the surgeon or attending physician.

\section{Deep SSI}

Infection occurs within 30 days after the operation if no implant is left in place or within 1 year if implant is in place, and the infection appears to be related to the operation and infection involves deep soft tissues (eg, fascial and muscle layers) of the incision and at least one of the following:

- Purulent drainage from the deep incision but not from the organ/ space component of the surgical site.

- A deep incision spontaneously dehisces or is deliberately opened by a surgeon when the patient has at least one of the following signs or symptoms: fever $\left(>38^{\circ} \mathrm{C}\right)$, localised pain or tenderness, unless the site is culture-negative.

- An abscess or other evidence of infection involving the deep incision is found on direct examination, during reoperation or by histopathological or radiological examination.

- Diagnosis of a deep incisional SSI by a surgeon or an attending physician.

SSI, surgical site infection.

antibiotic uses, open or percutaneous drain, and negative pressure wound therapy, and timing of SSI treatment.

- Burst abdomen is defined as a fascial wound defect combined with internal organ evisceration, which occurs within 30 days after the index operation causing mandatory repair of the fascial wound.

- Seroma is defined as fluid collection in the area of incision or serous leakage through the wound, which appears within 3 months after the index operation.

- Haematoma is defined as a collection of blood, appearing in the incision within 7 days after the index operation, for which evacuation is needed.

- Enterocutaneous fistula and chronic wound sinus, which are rare complications, will be observed for 12 months.

- Acute postoperative pain will be recorded on days 1 and 3 after surgery. The pain score will be measured in the range from 0 (no pain) to 10 (worst pain).

- Chronic pain is defined as pain in any degree persisting at the incisional scar. This outcome will be evaluated at 3, 6, 12, 18 and 24 months after surgery.

- Length of hospital stay will be recorded as the number of days spent on admission after the index operation.
- Abdominal wall closure time is time spent starting from abdominal wall closure to completing skin approximation. Time will be recorded in minutes.

- Mesh removal rate. Mesh removal, for any reason, will be counted.

- Cost. All cost data, including direct medical, direct non-medical, and indirect costs, will be retrieved in Thai baht. Discounting will be applied to adjust for the difference in the timing of costs and outcome.

\section{Consent}

Eligible patients will be invited to participate at the time when consent to operate is obtained and will be provided with information about the trial's objective, benefits, and risks of interventions (see online supplemental document 1). Patients will be informed that their decision will not influence treatment, and the right to withdraw from participation at any time is preserved. All patients will be provided with at least 15 min to make a decision. Informed consent will be obtained by the principal investigators or authorised research assistants and signed by the participant and the witness before randomisation.

\section{Randomisation and concealment}

Participants will be randomly allocated to either RM or PSC, with a ratio of $1: 1$, by the research assistant. Stratified block randomisation will be used to generate random sequence lists considering collaborating centres as strata, with varying block sizes of 4-8. The allocation sequence will be confidential and kept in the Central Data Management Unit at the Department of Clinical Epidemiology and Biostatistics, Ramathibodi Hospital. Sequential numbers will be concealed in envelopes and distributed to each centre. Randomisation and concealment will be undertaken by statisticians not participating in the trial. Allocated treatment will be revealed to the surgeon prior to abdominal wall closure.

\section{Blinding}

Patients and outcome assessors will be blinded to avoid bias in outcome assessment. Although surgeons who perform the operations cannot be blinded, they will not be involved in outcome assessment. Intervention instructions will be available to the surgical team and patients under emergency situations or as a result of complications where knowledge of the treatment allocation is necessary.

\section{Data management}

Research assistants will record anonymised data in CRF, which will be checked for accuracy by the principal investigators. Any uncertainty will be resolved by crosschecking primary data sources. The principal investigator (ATa) will check and approve all CRFs before data entry. Web or onsite databases will be constructed following the structure of CRFs. A data quality control program will be developed for data entry. Data entry will be validated by two independent staff members to minimise error. Data access will be password protected and restricted to the principal investigator (ATa) and authorised research 


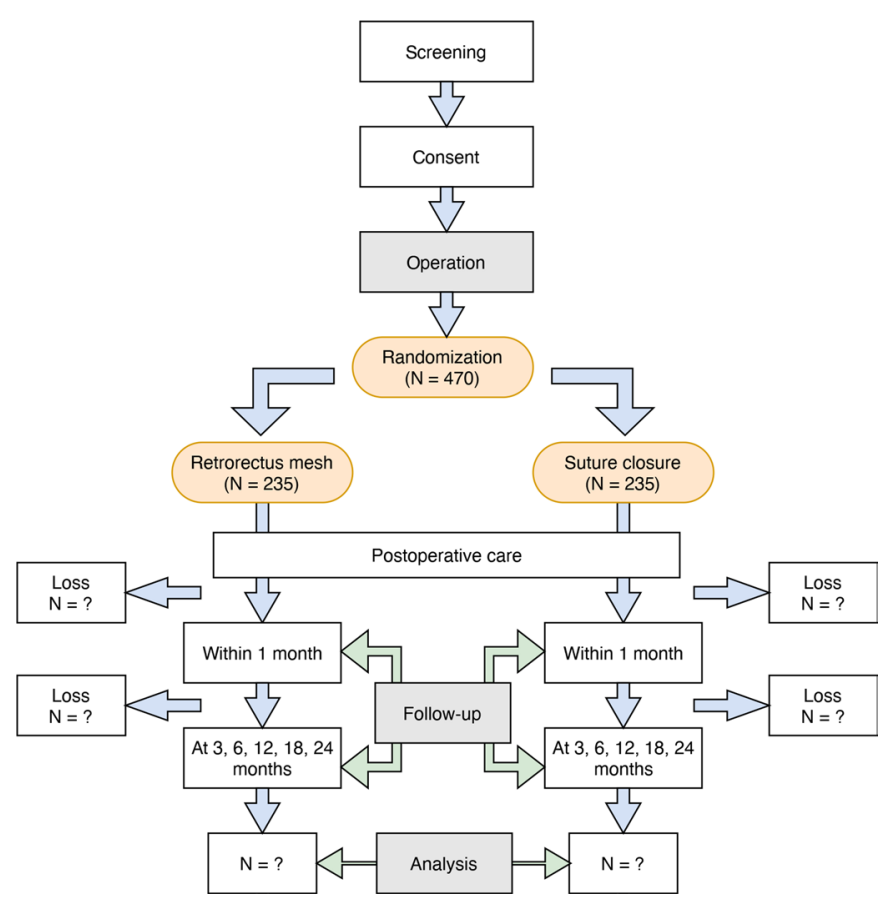

Figure 3 Consolidated Standards of Reporting Trials flow diagram.

assistants. Automated regular data backup will limit data loss. All paper documents will be securely stored in a locked cabinet. All data will be destroyed 5 years postpublication.

\section{Sample size determination}

A meta-analysis was applied to pool five RCTs of RM versus PSC, ${ }^{18-22}$ which demonstrated approximately $70 \%$ lower IH risk by RM. As such, we estimate a risk reduction of at least $50 \%$ should be clinically relevant. Postoperative emergency surgery incidence of IH from five studies ${ }^{9-13}$ ranged between $16 \%$ and $33 \%$, compared with $7 \%$ at Ramathibodi Hospital in 2019. A pooled IH incidence of $19 \%$ was estimated from previous studies and Ramathibodi Hospital. Type I error and the power of the test will be 0.05 and 0.8 , respectively. Using a randomisation ratio of 1:1, a sample size of 424 subjects is estimated. Considering a potential loss to follow-up of $10 \%, 470$ participants will be recruited over an enrolment period of 18-24 months (figure 3).

\section{Statistical analysis}

Categorical variables will be described by frequency and percentage, whereas continuous variables will be reported as mean and $\mathrm{SD}$ or median and range where appropriate. The balance of covariables between the two intervention groups will be explored and compared using $\chi^{2} /$ Fisher's exact and independent Student's t-test/ quantile-regression for categorical and continuous variables, respectively.

The incidence of IH, along with $95 \%$ CIs, will be estimated separately by the intervention groups; a relative treatment effect will be then assessed by estimate RR and risk difference (RD). In addition, the number needed to treat, along with the $95 \% \mathrm{CI}$, will be estimated accordingly. In case that randomisation could not work well (or in other words, covariables are unbalanced between the intervention and control groups), these RRs and RDs will be estimated using a bivariate regression analysis with adjustment for covariables.

\section{Protocol violation}

Protocol violation may result from mistaken treatment delivery, or surgeons may deliver another intervention, not the assigned intervention to the subject. Protocol violations will be considered using three approaches: intention to treat (ITT), per protocol (PP) and as treated (AS). These analyses will be applied for all major outcomes (ie, IH and SSI), but only ITT will be used for the secondary outcomes.

The ITT analyses patients in the groups to which they are randomised, regardless of actual treatment. The PP analysis will consider only patients who adhere to their allocated intervention and excludes those patients who do not receive their allocated intervention after randomisation. The AS analysis will consider the actual received intervention, regardless of randomisation, with adjustment of covariables at baseline using a counterfactual approach by an instrumental variable (IV) regression. ${ }^{23} 24$ For this, the assigned intervention will be considered as the IV, while the actual received intervention will be an endogenous variable. Two-stage bivariate probit or a logistic model, where appropriate, will be applied to estimate the effect of the intervention with adjustment for covariables.

\section{Imputation}

We will manually search for missing data in case record forms and medical records. If such data cannot be retrieved, missing data will be assumed to be missing at random, and imputation will be performed. The missing data will be regressed on complete data using linear truncated/interval or logistic regression with 10 imputations, depending on the variable type. The fraction of missing information and relative variance increase will be calculated as indices to assess imputation performance.

\section{Cost-effectiveness analysis}

Analyses will be based on societal and provider perspectives. The time horizon will be 24 months. Therefore, discounting will be applied to all costs. IH RD ( $\Delta$ effectiveness) and cost difference ( $\Delta$ cost) between SM and PSC will be calculated. The incremental cost-effectiveness ratio (ICER) will be computed by dividing $\Delta$ cost by $\Delta$ effectiveness.

ICER $=\Delta$ cost $/ \Delta$ effectiveness .

ICER and its replications from 1000 Monte Carlo simulations will be plotted on a cost-effectiveness plane according to different willingness-to-pay thresholds. The cost-effectiveness acceptability curve will be constructed as a function of willingness-to-pay thresholds. The net 
monetary benefit (NMB) will be computed at each threshold based on the following equation:

$\mathrm{NMB}=\mathrm{R} \cdot \Delta$ effectiveness $-\Delta$ cost,

where $\mathrm{R}$ is the willingness-to-pay threshold.

One-way sensitivity analyses will be performed where appropriate. Results from a series of one-way sensitivity analyses will be presented in a tornado diagram.

\section{Interim analysis}

Two additional analyses will be undertaken by an independent statistician, blinded to treatment allocation, before enrolment is completed. These analyses will evaluate the safety of the mesh placement and influence on short-term SSI rate when $50 \%$, and $75 \%$ of participants have enrolled or overall deep SSI is $5 \%$ or higher; this will be undertaken by the data safety and monitoring board (DSMB). To avoid inflation of type I error, the alphaspending function, proposed by O'Brien and Fleming, ${ }^{25}$ will be used for $\mathrm{p}$ value adjustment. After all participants have been enrolled, analyses for all interest outcomes will be completed.

\section{Analysis for early data release}

Early data release would provide useful information to the science community. Thus, additional analysis will be conducted after complete enrolment and 12-month follow-up in all participants. Secondary outcomes, including SSI, seroma, haematoma, chronic pain and fistula, will be analysed and reported. Multiplecomparison adjustment is not required for this analysis because data will not affect the release and follow-up protocol. After all participants are enrolled and followed up for 24 months (ie, the planned follow-up period for IH outcome), the analyses defined will be repeated, and the trial will be concluded.

\section{Monitoring and safety}

The proposed DSMB will consist of surgeons, epidemiologists and biostatisticians independent of the RCT. DSMB meetings will be organised every 6 months and when thresholds are reached. All adverse events (eg, SSI, seroma, haematoma, etc) and relevant source documents for data validation will be reported and accessible to DSMB in a confidential manner.

\section{Early termination of the trial}

In consultation with the steering committee and the DSMB, the investigators may terminate the trial at the recommendation of the DSMB for a number of possible scenarios:

- High rate of serious complications, for example, significantly higher SSI rate in the intervention group.

- Rate of enrolment was poor.

- External independent evidence that suggested the trial was unethical or where new research evidence is released.
ETHICS AND DISSEMINATION

This study will be conducted following the Declaration of Helsinki and the International Conference on Harmonisation-Good Clinical Practice E6 recommendation. The enrolment in each study site will commence after the approval of the local ethics committee. The study has been approved by the ethics committee of Ramathibodi Hospital, which is the main institute (MURA2020/1478), and also the collaboration cite including Vajira Hospital (COA164/2563). Written informed consent must be obtained before participation. Adverse events will be treated according to the standard of care and reimbursed according to Thai healthcare reimbursement schemes. No biological specimens will be collected as part of this trial.

Results of this trial will be published in a peer-reviewed journal. Only summary data, which cannot identify individual participants, will be presented in manuscripts. Datasets will be anonymised and made available for sharing among researchers.

\section{DISCUSSION}

An IH incidence of $20 \%$ following abdominal surgery ${ }^{1}$ commonly results in complications and poorer quality of life ${ }^{26}$ necessitating hernia repair. Unfortunately, the recurrence rate is high, regardless of the repair technique $(7.3 \%-21.1 \%),{ }^{27-30}$ emphasising the importance of hernia prophylaxis. A cost analysis of IH repair in France concluded that 4 million euros could be saved per year by reducing $\mathrm{IH}$ incidence by $5 \% .^{31}$

A significant body of evidence exists demonstrating the efficacy of mesh augmentation in hernia prophylaxis, especially in high-risk patients. ${ }^{18-21 ~ 32-38}$ Emergency operation is another risk factor for IH; however, none of the published RCTs investigated the efficacy of prophylactic mesh placement specifically in this group of patients. This RCT aims to examine the effect of RM augmentation on IH occurrence after emergency/urgent midline laparotomy.

Even though RM may be less effective than onlay mesh for hernia prevention, it results in less wound-related complications. ${ }^{8}$ Thus, RM augmentation was selected as the intervention of interest. The Preemer trial is currently assessing the efficacy of RM placement in emergency laparotomy, although the technique used differs from that proposed here. ${ }^{39}$ The Preemer trial ${ }^{39}$ uses a self-gripping mesh instead of the commonly used polypropylene mesh. Considering costs, the plain lightweight polypropylene mesh might be more easily adopted for prophylaxis.

The proposed trial has several strengths. The sample size is relatively large compared with previous studies of IH prophylaxis. ${ }^{18-2032-38}$ Moreover, participants, outcome assessors and data analysts will be blinded to minimise potential bias. Furthermore, the primary outcome will be assessed at 24 months, in line with the European Hernia Society guidelines, ${ }^{40}$ and all related costs will be evaluated alongside clinical outcomes providing measures of costeffectiveness to inform policy decisions. 
This trial will have some challenges. The intervention requires significant surgical expertise, although this is ameliorated by technique standardisation and appropriate training procedures. Protocol violations may occur but will be considered under ITT, PP and AS. In addition, intervention adherence will be evaluated using IV regression analysis, which maintains the benefits of the randomisation process.

In conclusion, this trial is a prospective, randomised, open, blinded endpoint design that will inform hernia prevention, especially in emergency/urgency settings.

\section{Author affiliations}

${ }^{1}$ Surgery, Navamindradhiraj University, Bangkok, Thailand

${ }^{2}$ Clinical Epidemiology and Biostatistics, Faculty of Medicine Ramathibodi Hospital, Mahidol University, Bangkok, Thailand

${ }^{3}$ Surgery, Faculty of Medicine Ramathibodi Hospital, Mahidol University, Bangkok, Thailand

${ }^{4}$ Centre for Public Health, Faculty of Medicine Health and Life Sciences, Queen's University Belfast, Belfast, UK

${ }^{5}$ Centre for Clinical Epidemiology and Biostatistics, Hunter Medical Research Institute, School of Medicine and Public Health, The University of Newcastle, New Lambton, New South Wales, Australia

Acknowledgements This manuscript is a part of ATa's training in the international PhD programme (Clinical Epidemiology) at the Department of Clinical Epidemiology and Biostatistics, Faculty of Medicine Ramathibodi Hospital, and Faculty of Graduate Studies, Mahidol University, Bangkok, Thailand. This protocol is a part of his dissertation.

Contributors ATa is the principal investigator. ATa, ST and PS designed the study. ATh provided help in statistical design. ATa drafted the manuscript. ATh, PN, GMK and JA critically revised the study design and the manuscript. The entire project will be supervised by PN, PS and ATh.

Funding The authors have not declared a specific grant for this research from any funding agency in the public, commercial or not-for-profit sectors.

Competing interests None declared.

Patient consent for publication Not required.

Provenance and peer review Not commissioned; externally peer reviewed.

Supplemental material This content has been supplied by the author(s). It has not been vetted by BMJ Publishing Group Limited (BMJ) and may not have been peer-reviewed. Any opinions or recommendations discussed are solely those of the author(s) and are not endorsed by BMJ. BMJ disclaims all liability and responsibility arising from any reliance placed on the content. Where the content includes any translated material, BMJ does not warrant the accuracy and reliability of the translations (including but not limited to local regulations, clinical guidelines, terminology, drug names and drug dosages), and is not responsible for any error and/or omissions arising from translation and adaptation or otherwise.

Open access This is an open access article distributed in accordance with the Creative Commons Attribution Non Commercial (CC BY-NC 4.0) license, which permits others to distribute, remix, adapt, build upon this work non-commercially, and license their derivative works on different terms, provided the original work is properly cited, appropriate credit is given, any changes made indicated, and the use is non-commercial. See: http://creativecommons.org/licenses/by-nc/4.0/.

\section{ORCID iDs}

Amarit Tansawet http://orcid.org/0000-0002-2040-7970

Suphakarn Techapongsatorn http://orcid.org/0000-0001-5373-7362

Gareth McKay http://orcid.org/0000-0001-8197-6280

John Attia http://orcid.org/0000-0001-9800-1308

\section{REFERENCES}

1 Rhemtulla IA, Messa CA, Enriquez FA, et al. Role of prophylactic mesh placement for laparotomy and stoma creation. Surg Clin North Am 2018;98:471-81.
2 Bhangu A, Fitzgerald JE, Singh P, et al. Systematic review and metaanalysis of prophylactic mesh placement for prevention of incisional hernia following midline laparotomy. Hernia 2013;17:445-55.

3 Nachiappan S, Markar S, Karthikesalingam A, et al. Prophylactic mesh placement in high-risk patients undergoing elective laparotomy: a systematic review. World J Surg 2013;37:1861-71.

4 Timmermans L, de Goede B, Eker HH, et al. Meta-analysis of primary mesh augmentation as prophylactic measure to prevent incisional hernia. Dig Surg 2013;30:401-9.

5 Borab ZM, Shakir S, Lanni MA, et al. Does prophylactic mesh placement in elective, midline laparotomy reduce the incidence of incisional hernia? A systematic review and meta-analysis. Surgery 2017;161:1149-63.

6 Wang X-C, Zhang D, Yang Z-X, et al. Mesh reinforcement for the prevention of incisional hernia formation: a systematic review and meta-analysis of randomized controlled trials. J Surg Res 2017;209:17-29.

7 Payne R, Aldwinckle J, Ward S. Meta-analysis of randomised trials comparing the use of prophylactic mesh to standard midline closure in the reduction of incisional herniae. Hernia 2017;21:843-53.

8 Tansawet A, Numthavaj P, Techapongsatorn S, et al. Mesh position for hernia prophylaxis after midline laparotomy: a systematic review and network meta-analysis of randomized clinical trials. Int J Surg 2020;83:144-51.

9 Mingoli A, Puggioni A, Sgarzini G, et al. Incidence of incisional hernia following emergency abdominal surgery. Ital J Gastroenterol Hepatol 1999;31:449-53.

10 Kurmann A, Barnetta C, Candinas D, et al. Implantation of prophylactic nonabsorbable intraperitoneal mesh in patients with peritonitis is safe and feasible. World J Surg 2013;37:1656-60.

11 Argudo N, Pereira JA, Sancho JJ, et al. Prophylactic synthetic mesh can be safely used to close emergency laparotomies, even in peritonitis. Surgery 2014;156:1238-44.

12 Saleem A-E-A, Abdallah H, Abdul Raheem O, et al. Rate of development of incisional hernia 1 year after urgent midline laparotomy. Al-Azhar Assiut Medical Journal 2016;14:59-66.

13 Basta MN, Kozak GM, Broach RB, et al. Can we predict incisional hernia?: development of a Surgery-specific Decision-Support interface. Ann Surg 2019;270:544-53.

14 Burns FA, Heywood EG, Challand CP, et al. Is there a role for prophylactic mesh in abdominal wall closure after emergency laparotomy? A systematic review and meta-analysis. Hernia 2020;24:441-7.

15 Lima HVG, Rasslan R, Novo FCF, et al. Prevention of fascial dehiscence with onlay prophylactic mesh in emergency laparotomy: a randomized clinical trial. J Am Coll Surg 2020;230:76-87.

16 Chan A-W, Tetzlaff JM, Altman DG, et al. Spirit 2013 statement: defining standard protocol items for clinical trials. Ann Intern Med 2013;158:200-7.

17 Mangram AJ, Horan TC, Pearson ML, et al. Guideline for prevention of surgical site infection, 1999. centers for disease control and prevention (CDC) hospital infection control practices Advisory Committee. Am J Infect Control 1999;27:97-132.

18 Strzelczyk JM, Szymański D, Nowicki ME, et al. Randomized clinical trial of postoperative hernia prophylaxis in open bariatric surgery. $\mathrm{Br}$ $J$ Surg 2006;93:1347-50.

19 Bevis PM, Windhaber RAJ, Lear PA, et al. Randomized clinical trial of mesh versus sutured wound closure after open abdominal aortic aneurysm surgery. Br J Surg 2010;97:1497-502.

20 Muysoms FE, Detry O, Vierendeels T, et al. Prevention of incisional hernias by prophylactic Mesh-augmented reinforcement of midline laparotomies for abdominal aortic aneurysm treatment: a randomized controlled trial. Ann Surg 2016;263:638-45.

21 Jairam AP, Timmermans L, Eker HH, et al. Prevention of incisional hernia with prophylactic onlay and sublay mesh reinforcement versus primary suture only in midline laparotomies (PRiMA): 2-year follow-up of a multicentre, double-blind, randomised controlled trial. Lancet 2017;390:567-76.

22 Pizza F, D'Antonio D, Arcopinto M, et al. Safety and efficacy of prophylactic resorbable biosynthetic mesh following midline laparotomy in clean/contemned field: preliminary results of a randomized double blind prospective trial. Hernia 2020;24:85-92.

23 Bagiella E, Karamlou T, Chang H, et al. Instrumental variable methods in clinical research. J Thorac Cardiovasc Surg 2015;150:779-82.

24 Höfler M. Causal inference based on counterfactuals. BMC Med Res Methodol 2005;5:28.

25 O'Brien PC, Fleming TR. A multiple testing procedure for clinical trials. Biometrics 1979;35:549-56.

26 van Ramshorst GH, Eker HH, Hop WCJ, et al. Impact of incisional hernia on health-related quality of life and body image: a prospective cohort study. Am J Surg 2012;204:144-50. 
27 Israelsson LA, Smedberg S, Montgomery A, et al. Incisional hernia repair in Sweden 2002. Hernia 2006;10:258-61.

28 Helgstrand F, Rosenberg J, Kehlet $\mathrm{H}$, et al. Nationwide prospective study of outcomes after elective incisional hernia repair. J Am Coll Surg 2013;216:217-28.

29 Kokotovic D, Bisgaard T, Helgstrand F. Long-Term recurrence and complications associated with elective incisional hernia repair. JAMA 2016;316:1575-82.

30 Pereira JA, López-Cano M, Hernández-Granados P, et al. Initial results of the National Registry of incisional hernia. Cir Esp 2016;94:595-602.

31 Gillion J-F, Sanders D, Miserez M, et al. The economic burden of incisional ventral hernia repair: a multicentric cost analysis. Hernia 2016;20:819-30.

32 Gutiérrez de la Peña C, Medina Achirica C, Domínguez-Adame E, et al. Primary closure of laparotomies with high risk of incisional hernia using prosthetic material: analysis of usefulness. Hernia 2003;7:134-6.

33 El-Khadrawy OH, Moussa G, Mansour O, et al. Prophylactic prosthetic reinforcement of midline abdominal incisions in high-risk patients. Hernia 2009;13:267-74.

34 Abo-Ryia MH, El-Khadrawy OH, Abd-Allah HS. Prophylactic preperitoneal mesh placement in open bariatric surgery: a guard against incisional hernia development. Obes Surg 2013;23:1571-4.
35 García-Ureña Miguel Ángel, López-Monclús J, Hernando LAB, et al. Randomized controlled trial of the use of a large-pore polypropylene mesh to prevent incisional hernia in colorectal surgery. Ann Surg 2015;261:876-81.

36 Glauser PM, Brosi P, Speich B, et al. Prophylactic intraperitoneal onlay mesh following midline Laparotomy-Long-Term results of a randomized controlled trial. World J Surg 2019;43:1669-75.

37 Caro-Tarrago A, Olona C, Millán M, et al. Long-term results of a prospective randomized trial of midline laparotomy closure with onlay mesh. Hernia 2019;23:335-40.

38 Kohler A, Lavanchy JL, Lenoir U, et al. Effectiveness of prophylactic intraperitoneal mesh implantation for prevention of incisional hernia in patients undergoing open abdominal surgery: a randomized clinical trial. JAMA Surg 2019;154:109-8.

39 Mäkäräinen-Uhlbäck E. Preemer Trial - Prophylactic Mesh Versus no Mesh in the Midline Emergency Laparotomy Closure for Prevention of Incisional Hernia: a Multi Center, Double-blind Randomized Controlled Trial, 2020. Available: https://clinicaltrials.gov/ct2/show/ NCT04311788

40 Muysoms FE, Antoniou SA, Bury K, et al. European hernia Society guidelines on the closure of abdominal wall incisions. Hernia 2015;19:1-24. 